\title{
Dual Homogeneous Forms and Varieties of Power Sums
}

\author{
Igor V. Dolgachev
}

\begin{abstract}
We review the classical definition of the dual homogeneous form of arbitrary even degree which generalizes the well-known notion of the dual quadratic form. Following the ideas of S. Mukai we apply this construction to the study of the varieties parametrizing representations of a homogeneous polynomial as a sum of powers of linear forms.
\end{abstract}

Mathematics Subject Classification (2000). Primary 14N15; Secondary 14J45, 15A72.

Keywords. Apolarity, homogeneous forms, sums of powers, Fano threefolds.

\section{Introduction}

A well-known theorem from linear algebra asserts that a nondegenerate quadratic form $F_{2}$ on a complex vector space $V$ of dimension $n+1$ can be written as a sum of $n+1$ squares of linear forms $l_{i}$. The linear forms $l_{i}$ 's considered as vectors in the dual space $V^{*}$ are mutually orthogonal with respect to the dual quadratic form $\check{F}_{2}$ on the space $V^{*}$. For more than hundred years it has been a popular problem for algebraists and geometers to search for a generalization of this construction to homogeneous forms $F_{d}$ on $V$ of arbitrary degree. It is known as the Waring problem or the canonical forms problem for homogeneous forms. The main object of the study is the variety of sums of powers $\operatorname{VSP}\left(F_{d} ; N\right)^{o}$ parametrizing all representations of $F_{d}$ as a sum of powers of $N$ linear forms. According to the traditional

Research supported in part by NSF Grant DMS 0245203. 
definition, $\operatorname{VSP}\left(F_{d} ; N\right)^{o}$ is the subset of the projective space $\mathbb{P} S^{N} V^{*}$ of homogeneous polynomials of degree $N$ on $V$ equal to the product of linear forms $l_{1} \ldots l_{N}$ such that $F_{d}=\sum l_{i}^{d}$. The zero sets $V\left(l_{i}\right)$ of the forms $l_{i}$ 's are hyperplanes in $\mathbb{P} V$, and the set $V\left(l_{1}\right), \ldots, V\left(l_{N}\right)$ was classically known as the polar polyhedron of $F_{d}$. Its faces $V\left(l_{i}\right)$ can be viewed as points in the dual space $\mathbb{P} V^{*}$, and the variety $\operatorname{VSP}\left(F_{d} ; N\right)^{o}$ is the subvariety of the symmetric power $\mathbb{P} V^{*(N)}$ of $\mathbb{P} V^{*}$ parametrizing the polar polyhedra of $F_{d}$. The varietes $\operatorname{VSP}\left(F_{d} ; N\right)^{o}$ were intensively studied in the classical algebraic geometry and the invariant theory in the works of A. Dixon, F. Palatini, T. Reye, H. Richmond, J. Rosanes, G. Scorza, A. Terracini, and others. However, the lack of techniques of higher dimensional algebraic geometry did not allow them to give any explicit construction of the varieties $\operatorname{VSP}\left(F_{d} ; N\right)^{o}$ or to study a possible compactification $\operatorname{VSP}\left(F_{d} ; N\right)$ of $\operatorname{VSP}\left(F_{d} ; N\right)^{o}$ (except in the case $n=1$ and a few cases where $\operatorname{VSP}\left(F_{2}, N\right)^{\circ}$ is a finite set of points). The first explicit construction of $\operatorname{VSP}\left(F_{d} ; N\right)^{o}$ was given by S. Mukai in the cases $(n, d, N)=(2,2,3),(2,4,6),(2,6,10)$ for a general polynomial $F_{d}$. He also constructed a smooth compactification $\operatorname{VSP}\left(F_{d} ; N\right)$ which turned out to be a Fano threefold in the first two cases and a K3 surface in the third case. By a different method, this result was proven later by K. Ranestad and F.-O. Schreyer [12]. Other explicit smooth compactifications of $\operatorname{VSP}(F ; N)$ are known only for general cubic polynomials in $n+1 \leq 6$ variables. If $n=2, \operatorname{VSP}(F ; 4)$ is isomorphic to the projective plane [3], if $n=3, \operatorname{VSP}(F ; 5)$ is the one-point set (this a classical result of Sylvester, see [14]), if $n=4, \operatorname{VSP}(F, 8)$ is a smooth 5-dimensional Fano variety and, if $n=5, \operatorname{VSP}(F, 10)$ is a holomorphic symplectic 4 -fold (see [8]). The construction of S. Mukai is very beautiful and employs a generalization of the notion of the dual quadratic form to forms of arbitrary even degree $d=2 k$. This generalization emerges from the classical theory of apolarity, a nowadays almost forgotten chapter in multilinear algebra. One associates to $F_{d}$ a quadratic form $\Omega_{F}$ on the symmetric power $S^{k} V$ of $V$ and, if this form is non-degenerate, the dual quadratic form $\check{\Omega}_{F} \in S^{2}\left(S^{k} V^{*}\right)$. The multiplication map $S^{2} S^{k} V^{*} \rightarrow S^{2 k} V^{*}$ defines the dual form $\check{F}$ of degree $d$ on $V^{*}$. A representation $F_{2 k}=l_{1}^{2 k}+\ldots+l_{N}^{2 k}$ can be viewed as a representation of $\Omega_{F}$ as a sum of squares of linear forms from $S^{k} V^{*}$ which happen to be $k$ th powers of linear forms. It follows from this that the minimal $N$ for which $\operatorname{VSP}\left(F_{2 k}, N\right)^{o} \neq \emptyset$ (which we call the Waring rank of $F_{d}$ ) is greater or equal to the rank of $\Omega_{F}$. The cases considered by Mukai are the cases where the equality takes place. 
The main goal of this paper is to fill in some of the details of Mukai's construction sketched in [10]. We make an effort to use the techniques of algebraic geometry as less as possible. A few original results can be found in the last section.

I am grateful to Kristian Ranestad for some comments on the paper and Michael Sagranoff for sending me his diploma paper.

\section{The apolarity pairing}

\subsection{Apolar forms}

Let $V$ be a complex vector space of dimension $n+1$ and $V^{*}$ be its dual vector space. We have a canonical duality pairing

$$
V \times V^{*} \rightarrow \mathbb{C}, \quad\langle v, l\rangle=l(v) .
$$

This can be naturally extended to a canonical perfect bilinear pairings

$$
S^{k} V \times S^{d} V^{*} \rightarrow S^{d-k} V^{*}, \quad(\Phi, F) \mapsto\langle\Phi, F\rangle
$$

between the symmetric powers of $V^{*}$ and $V$ (by definition, $S^{d-k} V^{*}=0$ if $d<k$ ). The easiest way to do this is via coordinates. Choose coordinates $t_{0}, \ldots, t_{n}$ in $V$ and the dual coordinates $\xi_{0}, \ldots, \xi_{n}$ in $V^{*}$ to identify the symmetric algebra $S^{\bullet} V^{*}\left(\right.$ resp. $S^{\bullet} V$ ) with the polynomial algebra $R=\mathbb{C}\left[t_{0}, \ldots, t_{n}\right]$ (resp. $\left.U=\mathbb{C}\left[\xi_{0}, \ldots, \xi_{n}\right]\right)$. Then $S^{d} V^{*}$ is identified with the space $R_{k}$ of degree $k$ homogeneous polynomials and we have a similar identification for $S^{k} V$. For any homogeneous polynomial $\Phi\left(\xi_{0}, \ldots, \xi_{n}\right) \in U_{k}$ consider the linear differential operator

$$
D_{\Phi}=\Phi\left(\partial_{0}, \ldots, \partial_{n}\right) .
$$

by replacing the variable $\xi_{i}$ with the operator $\partial_{i}=\frac{\partial}{\partial t_{i}}$. Now set, for any $\Phi \in S^{k} V$ and $\Phi \in S^{d} V^{*}$,

$$
\langle\Phi, F\rangle=D_{\Phi}(F) .
$$

We call this pairing the apolarity paring.

Taking $k=d$, we obtain the duality isomorphisms

$$
S^{d} V^{*} \cong\left(S^{d} V\right)^{*}, \quad S^{d} V \cong\left(S^{k} V^{*}\right)^{*} .
$$

The following properties of the apolarity pairing follow easily from the definition.

(P1) $\left\langle\Phi \Phi^{\prime}, F\right\rangle=\left\langle\Phi,\left\langle\Phi^{\prime}, F\right\rangle\right\rangle$;

$(\mathrm{P} 2)\left\langle v^{d}, F\right\rangle=F(v)$, for any $v \in V$. 
Fixing $F \in S^{d} V^{*}$ we get a linear map

$$
\operatorname{ap}_{F}^{k}: S^{k} V \rightarrow S^{d-k} V^{*}, \quad \Phi \mapsto D_{\Phi}(F) .
$$

It is called the apolarity map.

Definition 2.1. A homogeneous form $\Phi \in S^{k}(V)$ is called apolar to a homogeneous form $F \in S^{d}\left(V^{*}\right)$ if $D_{\Phi}(F)=0$, or, in other words, $\Phi \in \operatorname{Ker}\left(\operatorname{ap}_{F}^{k}\right)$. The linear space of apolar forms of degree $k$ is denoted by $\operatorname{AP}_{k}(F)$.

Lemma 2.1. Let us identify $S^{k} V$ with $\left(S^{k} V^{*}\right)^{*}$ by means of the apolarity paring. Then

$$
\operatorname{ap}_{F}^{k}\left(S^{k} V\right)^{\perp}=\operatorname{AP}_{d-k}(F) .
$$

Proof. By Property (P1), for any $\Phi_{k} \in S^{k} V$ and any $\Phi_{d-k}^{\prime} \in S^{d-k} V$, we have

$$
\begin{gathered}
\left\langle\Phi_{d-k}^{\prime}, \operatorname{ap}_{F}^{k}\left(\Phi_{k}\right)\right\rangle=\left\langle\Phi_{d-k}^{\prime},\left\langle\Phi_{k}, F\right\rangle\right\rangle=\left\langle\Phi_{d-k}^{\prime} \Phi_{k}, F\right\rangle \\
=\left\langle\Phi_{k},\left\langle\Phi_{d-k}^{\prime}, F\right\rangle\right\rangle=\operatorname{ap}_{F}^{d-k}\left(\Phi_{d-k}^{\prime}\right)\left(\Phi_{k}\right) .
\end{gathered}
$$

Thus, if $\left\langle\Phi_{d-k}^{\prime}, \operatorname{ap}_{F}^{k}\left(\Phi_{k}\right)\right\rangle=0$ for all $\Phi_{k}$ we get $\operatorname{ap}_{F}^{d-k}\left(\Phi_{d-k}^{\prime}\right)\left(\Phi_{k}\right)=0$ for all $\Phi_{k}$. By nondegeneracy of the apolarity pairing we get $\operatorname{ap}_{F}^{d-k}\left(\Phi_{d-k}^{\prime}\right)=0$, i.e. $\Phi_{d-k}^{\prime} \in \operatorname{AP}_{d-k}(F)$. The converse is proven in the same way.

\subsection{Quadratic forms}

If $d=2$, the space $S^{2} V^{*}$ is the space of quadratic forms on $V$. In coordinates, $Q \in S^{2} V^{*}$ is given by a symmetric matrix $A=\left(a_{i j}\right)$

$$
Q\left(t_{0}, \ldots, t_{n}\right)=\sum_{i, j=0}^{n} a_{i j} t_{i} t_{j}
$$

The apolarity map is

$$
\operatorname{ap}_{Q}^{1}: V \rightarrow V^{*}, \quad v \mapsto D_{v}(Q)=2 \sum_{0 \leq i \leq j=n} a_{i j} v_{i} t_{j}=\sum_{j=0}^{n} \frac{\partial Q}{\partial t_{j}}(v) t_{j} .
$$

Considered as a bilinear form $V \times V \rightarrow \mathbb{C}$, this is the polar symmetric bilinear form $b_{Q}$ associated to $Q$. We shall identify $\operatorname{ap}_{Q}^{1}$ with $b_{Q}$ via the equality

$$
b_{Q}(v, w)=\left\langle w, \operatorname{ap}_{Q}^{1}(v)\right\rangle .
$$

The quadratic form $Q$ is nondegenerate if and only if $\mathrm{ap}_{Q}^{1}$ is an invertible linear map. In this case, there exists a unique quadratic form $\check{Q}$ on $V^{*}$ such that

$$
b_{\breve{Q}}=b_{Q}^{-1} .
$$


This quadratic form is called the dual quadratic form of $Q$. By solving the linear system of equations

$$
\sum_{i=0}^{n} a_{i j} x_{i}=y_{j}, \quad j=0, \ldots, n,
$$

we obtain that

$$
\check{Q}=\sum_{i, j=0}^{n} a_{i j}^{*} \xi_{i} \xi_{j},
$$

where $\left(a_{i j}^{*}\right)=\operatorname{adj}(A)$ is the matrix of complementary minors of $A$.

Applying (2.7), we get

$$
b_{\check{Q}}\left(b_{Q}(v), b_{Q}(v)\right)=\left\langle b_{Q}(v), b_{\check{Q}}\left(b_{Q}(v)\right\rangle=\left\langle b_{Q}(v), v\right\rangle=b_{Q}(v, v) .\right.
$$

This shows that

$$
Q(v)=0 \Longleftrightarrow \check{Q}\left(b_{Q}(v)\right) .
$$

Thus the map $b_{Q}: V \rightarrow V^{*}$ sends the zero locus of the quadric $Q$ to the zero locus of the dual quadric. It follows from (2.6) that, for any nonzero $v \in Q^{-1}(0)$ the hyperplane $b_{Q}(v)^{\perp}$ is the tangent hyperplane of $Q^{-1}(0)$ at $v$. Thus the dual quadric $\check{Q} \subset \mathbb{P} V^{*}$ is the locus of tangent hyperplanes of the quadric $V(Q) \subset \mathbb{P} V$.

\subsection{Dual homogeneous forms of higher degree}

We would like to generalize the notion of the dual quadratic form to the case $d>2$. Such a generalization is known for even $d=2 k$. Let $F \in S^{2 k} V^{*}$ and

$$
\operatorname{ap}_{F}^{k}: S^{k} V \rightarrow S^{k} V^{*}
$$

be the apolarity map (2.5). Using (2.4), we can view this map as a symmetric bilinear form

$$
\Omega_{F}: S^{k} V \times S^{k} V \rightarrow \mathbb{C}, \quad \Omega_{F}\left(\Phi_{1}, \Phi_{2}\right)=\operatorname{ap}_{F}^{k}\left(\Phi_{1}\right)\left(\Phi_{2}\right)=\left\langle\Phi_{2}, \operatorname{ap}_{F}^{k}\left(\Phi_{1}\right)\right\rangle .
$$

Let us identify $\Omega_{F}$ with the associated quadratic form on $S^{k} V$ (the restriction of $\Omega_{F}$ to the diagonal). This defines a linear map

$$
\Omega: S^{2 k} V^{*} \rightarrow S^{2} S^{k} V^{*}, \quad F \mapsto \Omega_{F} .
$$

There is also a natural left inverse map of $\Omega$

$$
P: S^{2} S^{k} V^{*} \rightarrow S^{2 k} V^{*}
$$


defined by multiplication $S^{k} V^{*} \times S^{k} V^{*} \rightarrow S^{2 k} V^{*}$. All these maps are $\mathrm{GL}(V)$-equivariant and realize the linear representation $S^{2 k} V^{*}$ as a direct summand in the representation $S^{2} S^{k} V^{*}$.

To see the expression of $\Omega_{F}$ in terms of coordinates, let us write $F$ in the form

$$
F=\sum_{|\mathbf{i}|=d} \frac{d !}{\mathbf{i} !} a_{\mathbf{i}} t^{\mathbf{i}} .
$$

Consider a basis in $S^{k}(V)$ formed by monomials $\xi^{\mathbf{i}}$ and a basis in $S^{k} V^{*}$ formed by monomials $t^{\mathbf{i}}$ ordered lexigraphically. The matrix of $\mathrm{ap}_{F}^{k}$ with respect to these bases is called the $k$ th catalecticant matrix of $F$ and is denoted by $\operatorname{Cat}_{k}(F)$. Its entries $c_{\mathbf{u v}}$ are parametrized by pairs $(\mathbf{u}, \mathbf{v}) \in$ $\mathbb{N}^{k} \times \mathbb{N}^{k}$ with $|\mathbf{u}|=|\mathbf{v}|=k$. We have

$$
c_{\mathbf{u v}}=a_{\mathbf{u}+\mathbf{v}} .
$$

Considering $a_{\mathbf{i}}$ as independent variables $T_{\mathbf{i}}$, we obtain the definition of a general catalecticant matrix $\operatorname{Cat}_{k}(n+1)$. This is a square matrix of size $\operatorname{dim} S^{k} V=\left(\begin{array}{c}n+k \\ k\end{array}\right)$. For $n=1$ this matrix is known as a Hankel matrix. Similarly one can define a non-square catalecticant matrix which defines the apolarity map $S^{k} V \rightarrow S^{d-k} V^{*}$.

As in the case of quadratic forms, the quadratic form $\Omega_{F}$ on the space $S^{k} V$ is defined by the symmetric matrix $\operatorname{Cat}_{k}(F)$. In particular, $\Omega_{F}$ is a non-degenerate quadratic form if and only if $\operatorname{det} \operatorname{Cat}_{k}(F) \neq 0$.

Definition 2.2. A homogeneous form $F \in S^{2 k} V^{*}$ is called nondegenerate if $\Omega_{F}$ is a nondegenerate quadratic form on $S^{k} V$.

Theorem 2.3. Assume that $F \in S^{2 k} V^{*}$ is nondegenerate. There exists a unique homogeneous form $\check{F} \in S^{2 k} V$ such that

$$
\Omega_{\check{F}}=\check{\Omega}_{F} .
$$

Proof. We know that $\check{\Omega}(F)$ is defined by the cofactor matrix $\operatorname{adj}\left(\operatorname{Cat}_{k}(F)\right)=$ $\left(c_{\mathbf{u v}}^{*}\right)$ so that

$$
\check{\Omega}_{F}=\sum c_{\mathbf{u v}}^{*} \xi^{\mathbf{u}} \xi^{\mathbf{v}} .
$$

Let

$$
\check{F}=\sum_{|\mathbf{u}+\mathbf{v}|=2 k} \frac{d !}{(\mathbf{u}+\mathbf{v}) !} c_{\mathbf{u v}}^{*} \xi^{\mathbf{u}+\mathbf{v}} .
$$


Recall that the entries $c_{\mathbf{u v}}$ of the catalecticant matrix depend only on the sum of the indices. Thus the entries of the cofactor matrix $\operatorname{adj}\left(\operatorname{Cat}_{k}(F)\right)=$ $\left(c_{\mathbf{u v}}^{*}\right)$ depend only on the sum of the indices. For any $t^{\mathbf{i}} \in S^{k} V^{*}$, we have

$$
D_{t^{\mathrm{i}}}(\check{F})=\sum_{\mathbf{u}, \mathbf{v}, \mathbf{u}+\mathbf{v} \geq \mathbf{i}} \frac{d !}{(\mathbf{u}+\mathbf{v}) !} c_{\mathbf{u v}}^{*} \frac{(\mathbf{u}+\mathbf{v}) !}{(\mathbf{u}+\mathbf{v}-\mathbf{i}) !} \xi^{\mathbf{u}+\mathbf{v}-\mathbf{i}}=\sum_{\mathbf{j},|\mathbf{j}|=k} \frac{d !}{\mathbf{j} !} c_{\mathbf{i j}}^{*} \xi^{\mathbf{j}}
$$

This checks that the matrix of the linear map $S^{k} V^{*} \rightarrow S^{k} V$ defined by $\Omega_{\check{F}}$ is equal to the matrix $\operatorname{adj}\left(\operatorname{Cat}_{k}(F)\right)$. Thus the quadratic form $\Omega_{\breve{F}}$ is the dual of the quadratic form $\Omega_{F}$.

Recall that the locus of zeroes of the quadric $\check{Q}$ in $V^{*}$ is equal to the set of linear functions of the form $l=b_{Q}(v)$ such that $\langle v, l\rangle=0$. The same is true for the dual form $\check{F}$. Its locus of zeroes consists of linear forms $l$ such that $\Omega_{F}^{-1}\left(l^{k}\right) \in S^{k} V$ vanishes on $l$. The degree $k$ homogeneous form $\Omega_{F}^{-1}\left(l^{k}\right)$ is classically known as the anti-polar of $l$ (with respect to $F$ ).

\section{Sums of powers}

\subsection{The variety $\operatorname{VSP}(F ; N)$ of sums of powers}

For any $F \in S^{m} V^{*}$ we denote by $V(F)$ the zero locus of $F$ in the projective space $\mathbb{P} V \cong \mathbb{P}^{n}$. For any nonzero $v \in V$ we denote by $[v]$ the corresponding point $\mathbb{C} v$ in $\mathbb{P} V$. The same notation is used for the dual space $\mathbb{P} V^{*}$. Thus any linear form $l \in V^{*}$ defines a hyperplane $V(l)$ in $\mathbb{P} V$ and at the same time a point $[l]$ in $\mathbb{P} V^{*}$.

For any finite set of points $p_{1}, \ldots, p_{N}$ in $\mathbb{P} V$ we denote by $L_{d}\left(\mathbb{P} V, p_{1}, \ldots, p_{N}\right)$ the linear space of homogeneous forms $F$ of degree $d$ in $\mathbb{P} V$ such that $V(F)$ contains the points $p_{1}, \ldots, p_{N}$.

Definition 3.1. An unordered set of points $\left[l_{1}\right], \ldots,\left[l_{N}\right]$ in $\mathbb{P} V^{*}$ is called a polar $N$-polyhedron of $F \in S^{d} V^{*}$ if there exist some nonzero scalars $\lambda_{1}, \ldots, \lambda_{N}$ such that

$$
F=\lambda_{1} l_{1}^{d}+\ldots+\lambda_{N} l_{N}^{d},
$$

and moreover the powers $l_{i}^{d}$ are linearly independent in $S^{d} V^{*}$.

Lemma 3.1. A set $\mathcal{P}=\left\{\left[l_{1}\right], \ldots,\left[l_{N}\right]\right\}$ is a polar polyhedron of $F$ if and only if

$$
L_{d}\left(\mathbb{P} V^{*},\left[l_{1}\right], \ldots,\left[l_{N}\right]\right) \subset \mathrm{AP}_{d}(F)
$$

and the above inclusion is not true anymore if we delete any $\left[l_{i}\right]$ from $\mathcal{P}$. 
Proof. Observe that vanishing of $\Phi \in S^{d} V$ at $l_{i} \in V^{*}$ is equivalent to $\left\langle\Phi, l_{i}^{d}\right\rangle=0$. Therefore

$$
\left\langle l_{1}^{d}, \ldots, l_{N}^{d}\right\rangle^{\perp}=L_{d}\left(\mathbb{P} V^{*},\left[l_{1}\right], \ldots,\left[l_{N}\right]\right) .
$$

The forms $l_{i}^{d}$ are linearly independent in $S^{d} V^{*}$ if and only if

$$
\operatorname{dim}\left\langle l_{1}^{d}, \ldots, l_{N}^{d}\right\rangle^{\perp}=\operatorname{codim}\left(\left\langle l_{1}^{d}, \ldots, l_{N}^{d}\right\rangle, S^{d} V^{*}\right)=\operatorname{dim} S^{d} V^{*}-N .
$$

Since passing through a point impose one linear condition on the coefficients of a form, we see that the forms $l_{i}^{d}$ are linearly independent if and only if the points $\left[l_{i}\right]$ impose independent conditions on hypersurfaces of degree $d$.

Suppose the conditions of the lemma are satisfied. Then

$$
F \in \mathrm{AP}_{d}(F)^{\perp} \subset L_{d}\left(\mathbb{P} V^{*},\left[l_{1}\right], \ldots,\left[l_{N}\right]\right)^{\perp}=\left\langle l_{1}^{d}, \ldots, l_{N}^{d}\right\rangle .
$$

Thus $F$ is a linear combination of the powers $l_{i}^{d}$. If $l_{1}^{d}, \ldots, l_{N}^{d}$ are linearly dependent, $\langle\mathcal{P}\rangle=\left\langle\mathcal{P}^{\prime}\right\rangle$ for some proper subset $\mathcal{P}^{\prime}$ of $\mathcal{P}$. Then

$$
\operatorname{AP}_{d}(F)^{\perp} \subset L_{d}\left(\mathbb{P} V^{*},\left[l_{1}\right], \ldots,\left[l_{N}\right]\right)^{\perp}=\left\langle\mathcal{P}^{\prime}\right\rangle
$$

and hence $\left\langle\mathcal{P}^{\prime}\right\rangle \subset \operatorname{AP}_{d}(F)$ contradicting the assumption. Thus $\mathcal{P}$ is a polar polyhedron of $F$.

Conversely, suppose $\mathcal{P}$ is a polar polyhedron of $F$. Then $F \in\langle\mathcal{P}\rangle$ and hence $\langle\mathcal{P}\rangle^{\perp} \subset\langle F\rangle^{\perp}=\operatorname{AP}_{d}(F)$. We have seen already that the second condition follows from the linear independence of the powers $l_{i}^{d}$.

We denote by $\operatorname{VSP}(F ; N)^{o}$ the subset of the symmetric power

$$
\left(\mathbb{P} V^{*}\right)^{(N)}=\left(\mathbb{P} V^{*}\right)^{N} / S_{N}
$$

which consists of polar $N$-polyhedra of $F$. By a standard argument from algebraic geometry, Lemma 3.1 implies that $\operatorname{VSP}(F ; N)^{\circ}$ is a locally closed subset of $\left(\mathbb{P} V^{*}\right)^{(N)}$.

One can consider different compactifications of the set $\operatorname{VSP}(F ; N)^{o}$. For example, we can view a polar polyhedron $\mathcal{P}$ as a reducible hypersurface $V\left(l_{1} \cdots l_{N}\right)$ of degree $N$. Then $\operatorname{VSP}(F ; N)^{o}$ becomes a locally closed subset of $\mathbb{P}\left(S^{N} V^{*}\right)$ and we define $\operatorname{VSP}(F ; N)$ to be its closure in the projective space. Another approach is to view $\left(\mathbb{P} V^{*}\right)^{(N)}$ as the locus of reduced subschemes in the Hilbert scheme $\left(\mathbb{P} V^{*}\right)^{[N]}$ of 0-dimensional closed subschemes of $\mathbb{P} V^{*}$ of length $N$. Then we define $\operatorname{VSP}(F ; N)$ to be the closure of $\operatorname{VSP}(F ; N)^{o}$ in the Hilbert scheme. Points in the complementary set $\operatorname{VSP}(F ; N) \backslash \operatorname{VSP}(F ; N)^{o}$ can be called generalized polar polyhedra of $F$. In the case $n=1$, the two compactifications coincide and generalized polar polyhedra admit the following interpretation. Let $P \in S^{N} V^{*}$ be a binary 
form such that $V(P) \in \operatorname{VSP}(F ; N)$. Write $P$ as the product of linear forms $P=l_{1}^{k_{1}} \ldots l_{s}^{k_{s}}$ with $k_{1}+\ldots+k_{s}=N$. Then

$$
F=A_{1} l_{1}^{d-k_{1}+1}+\cdots+A_{s} l_{s}^{d-k_{s}+1}
$$

for some binary forms $A_{i}$ of degree $k_{i}-1$.

In the case when $F$ is a sufficiently general form, the assertion of Lemma 3.1 can be extended to the case of generalized polar polyhedra: a closed subscheme $Z \in\left(\mathbb{P} V^{*}\right)^{[N]}$ belongs to $\operatorname{VSP}(F ; N)$ if and only if the linear space $L_{d}\left(\mathbb{P} V^{*}, Z\right)$ of degree $d$ forms vanishing on $Z$ belongs to $\operatorname{AP}_{d}(F)$ and this is not true anymore for any proper closed subscheme of $Z$. This can be easily deduced from the fact that the limit ideal of the homogeneous ideal defining $Z$ is saturated (see [7], 5.2).

Proposition 3.2. Assume $n=2$. For general $F \in S^{d} V^{*}$ the variety $\operatorname{VSP}(F ; N)$ is either empty or a smooth irreducible variety of dimension $N(n+1)-\left(\begin{array}{c}n+d \\ d\end{array}\right)$.

Proof. We consider the closure of $\operatorname{VSP}(F ; N)^{\circ}$ in the Hilbert scheme $\left(\mathbb{P} V^{*}\right)^{[N]}$. Recall that $Z \in(\mathbb{P} V)^{[N]}$ is a generalized polar polyhedron of $F$ if and only if $F \in L_{d}\left(\mathbb{P} V^{*}, Z\right)^{\perp}$ but this is not true for any proper closed subscheme $Z^{\prime}$ of $Z$. Consider the incidence variety

$$
X=\left\{(Z, F) \in\left(\mathbb{P} V^{*}\right)^{[N]} \times S^{d} V^{*}: Z \in \operatorname{VSP}(F ; N)\right\} .
$$

It is known that the Hilbert scheme of 0-cycles of a nonsingular surface is nonsingular $([5])$. Thus $\left(\mathbb{P} V^{*}\right)^{[N]}$ is nonsingular. Let $U$ be the open subset of the first factor such that for any point $Z \in U, \operatorname{dim} L_{d}\left(\mathbb{P} V^{*}, Z\right)=$ $\operatorname{dim} S^{d} V-N$. The fibre of the first projection over $Z \in U$ is an open Zariski subset of the linear space $L_{d}\left(\mathbb{P} V^{*}, Z\right)^{\perp}$. This shows that $X$ is irreducible and nonsingular. The fibres of the second projection are the varieties $\operatorname{VSP}(F ; N)$. Thus for an open Zariski subset of $S^{d} V^{*}$ the varieties $\operatorname{VSP}(F ; N)$ are nonsingular or empty.

\subsection{The Waring rank of a homogeneous form}

Since any quadratic form $Q$ can be reduced to a sum of squares, one can characterize its rank as the smallest number $r$ such that

$$
Q=l_{1}^{2}+\ldots+l_{r}^{2}
$$

for some linear forms $l_{1}, \ldots, l_{r}$. 
Definition 3.3. Let $F \in S^{d} V^{*}$. Its Waring $\operatorname{rank} \operatorname{wrk}(F)$ is the smallest number $r$ such that

$$
F=l_{1}^{d}+\ldots+l_{r}^{d}
$$

for some linear forms $l_{1}, \ldots, l_{r}$.

Proposition 3.4. Let $\Omega_{F}$ be the quadratic form on $S^{k} V$ associated to $F \in$ $S^{2 k} V^{*}$. Then the Waring rank of $F$ is greater or equal than the rank of $\Omega_{F}$.

Proof. Suppose (3.2) holds with $d=2 k$. Since $\Omega_{F}$ is linear with respect to $F$, we have $\Omega_{F}=\sum \Omega_{l_{i}^{2 k}}$. If we choose coordinates such that $l_{i}$ is a coordinate function $t_{0}$, we easily compute the catalecticant matrix of $l_{i}^{2 k}$. It is equal to the matrix with 1 at the upper left corner and zero elsewhere. The corresponding quadratic form is equal to $\left(t_{0}^{k}\right)^{2}$. Thus $\Omega_{l_{i}^{2 k}}=\left(l_{i}^{k}\right)^{2}$ and we obtain

$$
\Omega_{F}=\sum_{i=1}^{r} \Omega_{l_{i}^{2 k}}=\sum_{i=1}^{r}\left(l_{i}^{k}\right)^{2} .
$$

Thus the Waring rank of $F$ is greater or equal than the rank of $\Omega_{F}$.

Corollary 3.5. Suppose $F$ is a nondegenerate form of even degree $2 k$, then

$$
\operatorname{wrk}(F) \geq\left(\begin{array}{c}
k+n \\
n
\end{array}\right) .
$$

A naive way to compute the Waring rank is by counting constants. Consider the map

$$
s:\left(V^{*}\right)^{r} \rightarrow \mathbb{C}^{\left(\begin{array}{c}
d+n \\
n
\end{array}\right)}, \quad\left(l_{1}, \ldots, l_{r}\right) \mapsto \sum l_{i}^{d} .
$$

If $r(n+1) \geq\left(\begin{array}{c}d+n \\ n\end{array}\right)$ one expects that this map is surjective and hence wrk $(F) \leq r$ for general $F$. Here "general" means that the coefficients of $F$ belong to an open Zariski subset of the affine space $\mathbb{C}\left(\begin{array}{c}d+n \\ n\end{array}\right)$. It is a remarkable result of J. Alexander and A. Hirschowitz [1] that this is "almost always" true. The only exceptional cases when it is false and the map $s$ fails to be surjective are the following cases:

- $n=2, d=2, r=2, \operatorname{wrk}(F)=3$;

- $n=2, d=4, r=5, \operatorname{wrk}(F)=6$;

- $n=3, d=4, r=9, \operatorname{wrk}(F)=10$;

- $n=4, d=3, r=7, \operatorname{wrk}(F)=8$;

- $n=4, d=4, r=14, \operatorname{wrk}(F)=15$;

Here we assume that $F$ is a general form. 
Proposition 3.6. Let $F$ be a general homogeneous form of even degree $2 k$. Then

$$
\operatorname{wrk}(F)>\operatorname{rank} \Omega_{F}
$$

except in the following cases, where the equality takes place.

- $k=1$;

- $n=1$;

- $n=2, k \leq 4$;

- $n=3, k=2$.

Proof. The first case is obvious. It follows from considering the map (3.3) that $\operatorname{wrk}(F) \geq\left(\begin{array}{c}n+2 k \\ n\end{array}\right) /(n+1)$. On the other hand the rank of $\Omega_{F}$ for general $F$ is equal to $\operatorname{dim} S^{k} V=\left(\begin{array}{c}n+k \\ n\end{array}\right)$.

We know that the case $n=1$ is not exceptional so that we can compute the Waring rank of $F$ by counting constants and get $\operatorname{wrk}(F)=k+1=$ rank $\Omega_{F}$.

If $n=2$, we get $\operatorname{wrk}(F) \geq(2 k+2)(2 k+1) / 6=(k+1)(2 k+1) / 3$ and rank $\Omega_{F}=\left(\begin{array}{c}k+2 \\ 2\end{array}\right)=(k+2)(k+1) / 2$. We have $(k+1)(2 k+1) / 3>$ $(k+2)(k+1) / 2$ if $k>4$. By Alexander-Hirschowitz's result,

$$
\operatorname{wrk}(F)= \begin{cases}6 & \text { if } \mathrm{k}=2, \\ 10 & \text { if } \mathrm{k}=3, \\ 15 & \text { if } \mathrm{k}=3\end{cases}
$$

This shows that $\operatorname{wrk}(F)=\operatorname{rank} \Omega_{F}$ in all these cases.

If $n=3$, we get

$$
\operatorname{wrk}(F) \geq(2 k+3)(2 k+2)(2 k+1) / 24>\left(\begin{array}{c}
k+3 \\
3
\end{array}\right)=(k+3)(k+2)(k+1) / 6
$$

unless $k=2$.

Finally, it is easy to see that for $n>3$

$$
\operatorname{wrk}(F) \geq\left(\begin{array}{c}
2 k+n \\
n
\end{array}\right) /(n+1)>\left(\begin{array}{c}
k+n \\
n
\end{array}\right)
$$

for $k>1$.

Definition 3.7. Two linear forms $l, m \in V^{*}$ are called conjugate with respect to a nondegenerate form $F \in S^{2 k} V^{*}$ if

$$
\Omega_{\check{F}}\left(l^{k}, m^{k}\right)=\check{F}\left(l^{k} m^{k}\right)=0 .
$$

The next result can be found in [9] (certainly used by classics, see, for example, [13]). 
Proposition 3.8. Suppose $F$ is given by (3.2), where the powers $l_{i}^{k}$ are linearly independent in $S^{k} V^{*}$. Then each pair $l_{i}, l_{j}$ is conjugate with respect to $F$.

Proof. It follows from computation of $\Omega_{F}$ in the proof of Proposition 3.4 that it suffices to check the assertion for quadratic forms. Choose a coordinate system such that $l_{i}=t_{0}, l_{j}=t_{1}$ and $F=t_{0}^{2}+t_{2}^{2}+\ldots+t_{n}^{2}$. Then $\check{F}=\xi_{0}^{2}+\ldots+\xi_{n}^{2}$, where $\xi_{0}, \ldots, \xi_{n}$ are dual coordinates. Now the assertion is easily checked.

\subsection{Mukai's skew-symmetric form}

Let $\omega \in \Lambda^{2} V$ be a skew-symmetric bilinear form on $V^{*}$. It admits a unique extension to a Poisson bracket $\{,\}_{\omega}$ on $S^{\bullet} V^{*}$ which restricts to a skewsymmetric bilinear form

$$
\{,\}_{\omega}: S^{k+1} V^{*} \times S^{k+1} V^{*} \rightarrow S^{2 k} V^{*} .
$$

Recall that a Poisson bracket on a commutative algebra $A$ is a skewsymmetric bilinear map $A \times A \rightarrow A,(f, g) \mapsto\{f, g\}$ such that its left and right partial maps $A \rightarrow A$ are derivations.

Let $F \in S^{2 k} V^{*}$ be a nondegenerate form and $\check{F} \in S^{2 k} V=\left(S^{2 k} V^{*}\right)^{*}$ be its dual form. For each $\omega$ as above define $\sigma_{\omega, F} \in \Lambda^{2}\left(S^{k+1} V\right)^{*}$ by

$$
\sigma_{\omega, F}(f, g)=\check{F}\left(\{f, g\}_{\omega}\right) .
$$

Theorem 3.9 (S. Mukai). Let $F$ be a nondegenerate form in $S^{2 k} V^{*}$. For any $\mathcal{P}=\left\{\left[l_{1}\right], \ldots,\left[l_{N}\right]\right\} \in \operatorname{VSP}(F ; N)^{o}$ let $E(\mathcal{P})$ be the linear span of the powers $l_{i}^{k+1}$ in $S^{k+1} V^{*}$. Then

(i) $E(\mathcal{P})$ is isotropic with respect to each form $\sigma_{\omega, F}$;

(ii) $\operatorname{ap}_{F}^{k-1}\left(S^{k-1} V\right) \subset E(\mathcal{P})$;

(iii) $\operatorname{ap}_{F}^{k-1}\left(S^{k-1} V\right)$ is contained in the radical of each $\sigma_{\omega, F}$.

Proof. To prove the first assertion it is enough to check that $\sigma_{\omega, F}\left(l_{i}^{k+1}, l_{j}^{k+1}\right)$ $=0$ for all $i, j$. We have

$$
\sigma_{\omega, F}\left(l_{i}^{k+1}, l_{j}^{k+1}\right)=\check{F}\left(\left\{l_{i}^{k}, l_{j}^{k}\right\}_{\omega}\right)=\check{F}\left(l_{i}^{k} l_{j}^{k}\right) \omega\left(l_{i}, l_{j}\right) .
$$

By Proposition 3.8, $\check{F}\left(l_{i}^{k} l_{j}^{k}\right)=\Omega_{\breve{F}}\left(l_{i}^{k}, l_{j}^{k}\right)=0$. This checks the first assertion.

For any $\Phi \in S^{k-1} V$,

$$
D_{\Phi}(F)=D_{\Phi}\left(\sum_{i=1}^{N} l_{i}^{2 k}\right)=\sum D_{\Phi}\left(l_{i}^{2 k}\right)=\frac{(2 k) !}{(k+1) !} \sum_{i=1}^{N} D_{\Phi}\left(l^{k-1}\right) l_{i}^{k+1} .
$$


This shows that $\operatorname{ap}_{F}^{k-1}\left(S^{k-1} V\right)$ is contained in $E(\mathcal{P})$. It remains to check that for any $\Phi \in S^{k-1} V, G \in S^{k+1} V^{*}$ and any $\omega \in \Lambda^{2} V$, one has $\sigma_{\omega, F}\left(D_{\Phi}(F), G\right)=0$. Choose coordinates $t_{0}, \ldots, t_{n}$ in $V$ and the dual coordinates $\xi_{0}, \ldots, \xi_{n}$ in $V^{*}$. The space $\Lambda^{2} V$ is spanned by the forms $\omega_{i j}=\xi_{i} \wedge \xi_{j}$. We have

$$
\begin{gathered}
\left\{D_{\Phi}(F), G\right\}_{\omega_{i j}}=D_{\xi_{i}}\left(D_{\Phi}(F)\right) D_{\xi_{j}}(G)-D_{\xi_{j}}\left(D_{\Phi}(F)\right) D_{\xi_{i}}(G) \\
=D_{\xi_{i} \Phi}(F) D_{\xi_{j}}(G)-D_{\xi_{j} \Phi}(F) D_{\xi_{i}}(G)=D_{\Phi \xi_{i}}(F) D_{\xi_{j}}(G)-D_{\Phi \xi_{j}}(F) D_{\xi_{i}}(G) .
\end{gathered}
$$

For any $A, B \in S^{k} V^{*}$,

$$
\check{F}(A B)=\Omega_{\check{F}}(A, B)=\left\langle\Omega_{F}^{-1}(A), B\right\rangle .
$$

Thus

$$
\begin{gathered}
\sigma_{\omega_{i j}, F}\left(D_{\Phi}(F), G\right)=\check{F}\left(D_{\Phi \xi_{i}}(F) D_{\xi_{j}}(G)-D_{\Phi \xi_{j}}(F) D_{\xi_{i}}(G)\right) \\
=\left\langle\Phi \xi_{i}, D_{\xi_{j}}(G)\right\rangle-\left\langle\Phi \xi_{j}, D_{\xi_{i}}(G)\right\rangle=D_{\Phi}\left(D_{\xi_{i} \xi_{j}}(G)-D_{\xi_{j} \xi_{i}}(G)\right)=D_{\Phi}(0)=0 .
\end{gathered}
$$

\subsection{The Mukai map}

Let $F \in S^{2 k} V^{*}$ be a nondegenerate form. We assume that $(k, n)$ is one of the exceptional cases from Proposition 3.6.

Let

$$
N_{k}=\operatorname{rank} \Omega_{F}=\left(\begin{array}{c}
n+k \\
n
\end{array}\right) .
$$

We know that $\operatorname{VSP}\left(F ; N_{k}\right)^{o} \neq \emptyset$ for general enough $F$.

Let $\mathcal{P} \in \operatorname{VSP}\left(F ; N_{k}\right)^{o}$ and

$$
\bar{E}(\mathcal{P})=\left\langle l_{1}^{k+1}, \ldots, l_{N_{k}}^{k+1}\right\rangle / \operatorname{ap}_{F}^{k-1}\left(S^{k-1} V\right) .
$$

It is a subspace of $W=S^{k+1} V^{*} / \operatorname{ap}_{F}^{k-1}\left(S^{k-1} V\right)$. By Lemma 2.1,

$$
W^{*}=\operatorname{ap}_{F}^{k-1}\left(S^{k-1} V\right)^{\perp} \cong \operatorname{AP}_{k+1}(F),
$$

hence

$$
W \cong \operatorname{AP}_{k+1}(F)^{*}
$$

and

$$
\bar{E}(\mathcal{P}) \subset \operatorname{AP}_{k+1}(F)^{*} .
$$

Lemma 3.2. (i) $\operatorname{dim} \mathrm{AP}_{k+1}(F)^{*}=\left(\begin{array}{c}n+k \\ n-1\end{array}\right)+\left(\begin{array}{c}n+k-1 \\ n-1\end{array}\right)$;

(ii) $\operatorname{dim} E(\mathcal{P})=N_{k}$;

(iii) $\operatorname{dim} \bar{E}(\mathcal{P})=\left(\begin{array}{c}n+k-1 \\ n-1\end{array}\right)$. 
Proof. (i) Since $F$ is nondegenerate, $\operatorname{Ker}\left(\operatorname{ap}_{F}^{k}\right)=\{0\}$, hence $\operatorname{Ker}\left(\operatorname{ap}_{F}^{k-1}\right)=$ $\{0\}$ and

$$
\operatorname{dim} \operatorname{ap}_{F}^{k-1}\left(S^{k-1} V\right)=\operatorname{dim} S^{k-1} V^{*}=\left(\begin{array}{c}
k-1+n \\
n
\end{array}\right) .
$$

This gives

$$
\begin{gathered}
\operatorname{dim} \operatorname{AP}_{k+1}(F)^{*}=\operatorname{dim} S^{k+1} V-\operatorname{dim} \operatorname{ap}_{F}^{k-1}\left(S^{k-1} V\right)=\left(\begin{array}{c}
n+k+1 \\
n
\end{array}\right)-\left(\begin{array}{c}
n+k-1 \\
n
\end{array}\right) \\
=\left(\begin{array}{c}
n+k \\
n-1
\end{array}\right)+\left(\begin{array}{c}
n+k-1 \\
n-1
\end{array}\right) .
\end{gathered}
$$

(ii) Let $\mathcal{P}=\left\{\left[l_{1}\right], \ldots,\left[l_{N_{k}}\right]\right\}$. We have to show that the forms $l_{1}^{k+1}, \ldots$, $l_{N_{k}}^{k+1}$ are linearly independent. This is equivalent to the equality

$$
\operatorname{dim} L_{k+1}\left(\mathbb{P} V^{*},\left[l_{1}\right], \ldots,\left[l_{N_{k}}\right]\right)=\left(\begin{array}{c}
k+1+n \\
n
\end{array}\right)-N_{k}=\left(\begin{array}{l}
k+n \\
n-1
\end{array}\right) .
$$

This is obvious in the case $n=1$. If $k=1$, the space $L_{2}\left(\mathbb{P} V^{*},\left[l_{1}\right], \ldots,\left[l_{N_{k}}\right]\right)$ is of expected dimension unless $l_{1}^{2}, \ldots, l_{N_{k}}^{2}$ are linearly dependent. In our case this is impossible because $F$ is nodegenerate.

Assume $n=2, k=2$ and $\operatorname{dim} L_{3}\left(\mathbb{P} V^{*},\left[l_{1}\right], \ldots,\left[l_{6}\right]\right)>4$. Since $\operatorname{AP}_{2}(F)$ $=\{0\}$, no conic passes through the 6 points. In particular, no 4 points are collinear. Take a conic $C=V(Q)$ through 5 points $\left[l_{1}\right], \ldots,\left[l_{5}\right]$ and 2 points $x_{1}, x_{2}$ such that each component of $C$ contains $\geq 4$ points (if $C$ is reducible we take $x_{1}$ to be the intersection point of the two line components). Since

$$
\operatorname{dim} L_{3}\left(\mathbb{P} V^{*},\left[l_{1}\right], \ldots,\left[l_{6}\right], x_{1}, x_{2}\right)>2,
$$

there exist 3 linearly independent cubic forms $\Phi_{i}$ such that $V(\Phi)$ has 7 common points with $C$. Applying Bézout's Theorem, we see that the cubics contain $C$, hence we get a 2 -dimensional space of lines passing through $\left[l_{6}\right]$. This is impossible.

Assume $n=2, k=3$ and $\operatorname{dim} L_{4}\left(\mathbb{P} V^{*},\left[l_{1}\right], \ldots,\left[l_{10}\right]\right)>5$. Again there are no cubics through the ten points. In particular, no 5 points are collinear, and no 8 points are on a conic. We argue as before. Choose a cubic $C$ through the points $\left[l_{i}\right], i \neq 10$, and 3 points $x_{1}, x_{2}, x_{3}$ such that at least 5 points lie on each irreducible component of $C$. Since $\operatorname{dim} L_{4}\left(\mathbb{P} V^{*},\left[l_{1}\right], \ldots\right.$, $\left.\left[l_{10}\right], x_{1}, x_{2}, x_{3}\right)>2$, there exist 3 linearly independent quartic forms $\Phi_{i}$ such that $V(\Phi)$ has 13 common points with $C$. Applying Bézout's Theorem, we see that the quartics contain $C$, hence we get a 2 -dimensional space of lines passing through $\left[l_{10}\right]$. This is impossible.

A similar argument checks the assertion in the case $n=2, k=4$.

The last case $n=3, k=2$ needs to apply more techniques from algebraic geometry which we allow ourselves to use here. So, we skip the proof. 
(iii) We have

$$
\operatorname{dim} \bar{E}(\mathcal{P})=N_{k}-\operatorname{dim} \operatorname{ap}_{F}^{k-1}\left(S^{k-1} V\right)=\left(\begin{array}{c}
n+k \\
n
\end{array}\right)-\left(\begin{array}{c}
n+k-1 \\
k
\end{array}\right)=\left(\begin{array}{c}
n+k-1 \\
n-1
\end{array}\right) .
$$

We denote by $G(a, E)$ the Grassmannian of $a$-dimensional subspaces of a linear space $E$. Consider the regular map (we call it the Mukai map)

$$
\mathrm{Mu}: \operatorname{VSP}\left(F ; N_{k}\right)^{o} \rightarrow G\left(\left(\begin{array}{c}
n+k-1 \\
k
\end{array}\right), \operatorname{AP}_{k+1}(F)^{*}\right), \quad \mathcal{P} \mapsto \bar{E}(\mathcal{P}) .
$$

Proposition 3.10. The Mukai map is injective.

Proof. Suppose $\mathrm{Mu}\left(\left[l_{1}\right], \ldots,\left[l_{N_{k}}\right]\right)=\mathrm{Mu}\left(\left[m_{1}\right], \ldots,\left[m_{N_{k}}\right]\right)$. Then

$$
\left\langle l_{1}^{k+1}, \ldots, l_{N_{k}}^{k+1}\right\rangle=\left\langle m_{1}^{k+1}, \ldots, m_{N_{k}}^{k+1}\right\rangle,
$$

hence

$$
L_{k+1}\left(\mathbb{P} V^{*},\left[l_{1}\right], \ldots,\left[l_{N_{k}}\right]\right)=L_{k+1}\left(\mathbb{P} V^{*},\left[m_{1}\right], \ldots,\left[m_{N_{k}}\right]\right) .
$$

Without loss of generality we may assume that $\left[l_{1}\right]$ is not equal to any $\left[m_{i}\right]$. Since $\operatorname{dim} L_{k}\left(\mathbb{P} V^{*},\left[l_{2}\right], \ldots,\left[l_{N_{k}}\right]\right)>0$ we can find a degree $k$ form $\Phi$ vanishing at the last $N_{k}-1$ points. Let $L \in V$ be a linear form on $V^{*}$ which vanishes at $\left[l_{1}\right]$ but does not contain any of the points $\left[m_{1}\right], \ldots,\left[m_{N_{k}}\right]$. Since $L \Phi \in L_{k+1}\left(\mathbb{P} V^{*},\left[l_{1}\right], \ldots,\left[l_{N_{k}}\right]\right)$, we obtain that $L \Phi$ vanishes at any $\left[m_{i}\right]$, hence $\Phi$ vanishes at any $\left[m_{i}\right]$. Thus $\operatorname{AP}_{k}(F) \neq\{0\}$ which contradicts the nondegeneracy of $F$.

Therefore we may assume that $\left[l_{1}\right]=\left[m_{1}\right]$. Now if $\left[l_{2}\right] \neq\left[m_{i}\right]$ for any $i \geq$ 2 , we repeat the argument replacing $l_{1}$ with $l_{2}$ and obtain a contradiction. Proceeding in this way we show that the sets $\mathcal{P}$ and $\mathcal{P}^{\prime}$ coincide.

Remark 3.11. The same proof works for generalized polar polyhedra and general polynomial $F$. The analog of equality (3.7) is the equality $L_{k+1}\left(\mathbb{P} V^{*}, Z\right)=L_{k+1}\left(\mathbb{P} V^{*}, Z^{\prime}\right)$ of the spaces of polynomials of degree $k+1$ vanishing on $Z$ and $Z^{\prime}$. Suppose $Z \neq Z^{\prime}$. Choose a subscheme $Z_{0}$ of $Z$ of length $N_{k}-1$ which is not a subscheme of $Z^{\prime}$. Since $\operatorname{dim} L_{k}\left(\mathbb{P} V^{*}, Z_{0}\right) \geq$ $\left(\begin{array}{c}n+k \\ k\end{array}\right)-N_{k}+1>0$, we can find a nonzero $\Phi \in L_{k}\left(\mathbb{P} V^{*}, Z_{0}\right)$. The sheaf $\mathcal{I}_{Z} / \mathcal{I}_{Z_{0}}$ is concentrated at one point $x$ and is annihilated by the maximal ideal $\mathfrak{m}_{x}$. Thus $\mathfrak{m}_{x} \mathcal{I}_{Z_{0}} \subset \mathcal{I}_{Z}$. Let $L$ be a linear form vanishing at $x$ but not vanishing at any subscheme of $Z^{\prime}$. This implies that $L \Phi \in L_{k+1}\left(\mathbb{P} V^{*}, Z\right)=$ $L_{k+1}\left(\mathbb{P} V^{*}, Z^{\prime}\right)$ and hence $\Phi \in L_{k}\left(\mathbb{P} V^{*}, Z^{\prime}\right)$ contradicting the nondegeneracy of $F$. Using more techniques from algebraic geometry one can also prove that the Mukai map is a closed embedding. 
Recall that we have a linear map

$$
\Lambda^{2} V \rightarrow \Lambda^{2} S^{k+1} V=\left(\Lambda^{2} S^{k+1} V^{*}\right)^{*}, \quad \omega \mapsto \sigma_{\omega, F} .
$$

Since, $\operatorname{ap}_{F}^{k-1}\left(S^{k-1}\right)$ is contained in the radical of each $\sigma_{\omega, F}$ this map defines an injective map

$$
\Lambda^{2} V \rightarrow \Lambda^{2} \mathrm{AP}_{k+1}(F) .
$$

Let $\mathcal{N} \subset \Lambda^{2} \mathrm{AP}_{k+1}(F)$ be the image of this map. This is a linear subspace of the space of 2 -forms on $\operatorname{AP}_{k+1}(F)^{*}$. For each $\mathcal{P} \in \operatorname{VSP}\left(F ; N_{k}\right)$, the subspace $\bar{E}(\mathcal{P})$ is isotropic with respect to any $\sigma_{\omega, F}$. Let

$$
G\left(\left(\begin{array}{c}
n+k-1 \\
k
\end{array}\right), \operatorname{AP}_{k+1}(F)^{*}\right)_{\mathcal{N}} \subset G\left(\left(\begin{array}{c}
n+k-1 \\
k
\end{array}\right), \operatorname{AP}_{k+1}(F)^{*}\right)
$$

be the subvariety of the Grassmannian which consists of isotropic subspaces with respect to all 2 -forms from $\mathcal{N}$. Recall that a Grassmannian $G=G(s, E)$ admits a tautological exact sequence of vector bundles

$$
0 \rightarrow \mathcal{S}_{G} \rightarrow E_{G} \rightarrow \mathcal{Q}_{G} \rightarrow 0,
$$

where $E_{G}$ is the trivial bundle with fibre $E$, and $\mathcal{S}_{G}$ is the universal subbundle whose fibre at a point $x \in G$ is the linear $s$-dimensional subspace of $E$ corresponding to $x$. A 2-form $\alpha$ on $E$ defines, by restriction, a section $\sigma_{\alpha}$ of of $\Lambda^{2} \mathcal{S}_{G}$. Its set of zeroes $Z\left(\sigma_{\alpha}\right)$ is a closed subvariety of $G$ of codimension $\leq\left(\begin{array}{l}s \\ 2\end{array}\right)$ and the equality holds for a Zariski open subset (maybe empty) of sections (see [6]). It follows that for any $t$-dimensional subspace $L$ of sections, the set of common zeroes is of codimension $\leq t\left(\begin{array}{l}s \\ 2\end{array}\right)$, and the equality holds for a Zariski open subset of the Grassmann variety of $t$-dimensional spaces of sections.

Returning to our case, we see that

$$
\mathrm{Mu}\left(\operatorname{VSP}\left(F ; N_{k}\right)\right) \subset G\left(\left(\begin{array}{c}
n+k-1 \\
k
\end{array}\right), \operatorname{AP}_{k+1}(F)^{*}\right)_{\mathcal{N}} .
$$

The dimension of the left-hand side is equal to

$$
\begin{aligned}
& \operatorname{dim} \operatorname{VSP}\left(F ; N_{k}\right)=(n+1)\left(\begin{array}{c}
n+k \\
n
\end{array}\right)-\left(\begin{array}{c}
n+2 k \\
n
\end{array}\right) \\
& = \begin{cases}1 & \text { if } n=1, \\
\left(\begin{array}{c}
n+1 \\
2
\end{array}\right) & \text { if } k=1, \\
3 & \text { if } n=2, k=2, \\
2 & \text { if } n=2, k=3, \\
0 & \text { if } n=2, k=4, \\
5 & \text { if } n=3, k=2 .\end{cases}
\end{aligned}
$$


It is known that $\operatorname{dim} G(a, E)=a(\operatorname{dim} E-a)$. In our case

$$
\operatorname{dim} G\left(\left(\begin{array}{c}
n+k-1 \\
k
\end{array}\right), \operatorname{AP}_{k+1}(F)^{*}\right)=\left(\begin{array}{c}
n+k-1 \\
n-1
\end{array}\right)\left(\begin{array}{l}
n+k \\
n-1
\end{array}\right) .
$$

It follows from above that the expected dimension of $G\left(\left(\begin{array}{c}n+k-1 \\ k\end{array}\right), \mathrm{AP}_{k+1}^{*}\right)_{\mathcal{N}}$ is equal to $\operatorname{dim} \operatorname{VSP}\left(F ; N_{k}\right)$ in the cases $n=1$ and $n=2$. In all other cases, it is strictly less than $\operatorname{dim} \operatorname{VSP}\left(F ; N_{k}\right)$.

We already know that in the case $n=1$,

$$
\operatorname{VSP}(F, k+1)=\mathbb{P}^{1} \cong G\left(1, \operatorname{AP}_{k+1}(F)\right)_{\mathcal{N}}=G\left(1, \operatorname{AP}_{k+1}(F)\right) .
$$

The following theorem of Mukai says that the same is true for $n=2, k \leq 4$.

Theorem 3.12. (S. Mukai) Let $F_{d} \in S^{d} V^{*}$ be a general polynomial of degree $d=2 k$. Assume that $n=2, k \leq 4$. Then

$$
\operatorname{VSP}\left(F ;\left(\begin{array}{c}
k+2 \\
2
\end{array}\right)\right) \cong G\left(k+1, \operatorname{AP}_{k+1}(F)^{*}\right)_{\mathcal{N}} .
$$

(i) The variety $\operatorname{VSP}\left(F_{2} ; 3\right)$ is a smooth Fano 3-fold (of genus 21 and index 2);

(ii) The variety $\operatorname{VSP}\left(F_{4} ; 6\right)$ is a smooth Fano 3-fold (of genus 12 and index 1);

(iii) The variety $\operatorname{VSP}\left(F_{6} ; 10\right)$ is a smooth $K 3$ surface;

(iv) The variety $\operatorname{VSP}\left(F_{8} ; 15\right)$ is a set of 16 points.

Proof. By Proposition 3.2 the varieties $\operatorname{VSP}\left(F ;\left(\begin{array}{c}k+2 \\ 2\end{array}\right)\right)$ are smooth of the asserted dimension. Let $\mathcal{S}_{G}$ be the tautological vector bundle of rank $k+1$ on

$$
G\left(k+1, \operatorname{AP}_{k+1}(F)^{*}\right) \cong \begin{cases}G(2,5) & \text { if } k=1, \\ G(3,7) & \text { if } k=2, \\ G(4,9) & \text { if } k=3, \\ G(5,11) & \text { if } k=4 .\end{cases}
$$

A basis of a 3 -dimensional space $\mathcal{N}$ of sections of $\mathcal{E}=\Lambda^{2} \mathcal{S}_{G}^{*}$ defines a section $s$ of the vector bundle $\mathcal{E}^{\oplus 3}$. It is known that the vector bundle $\mathcal{E}$ is generated by global sections. By Bertini's Theorem for sections of vector bundles (see [11]) the zero locus $Z(s)$ of its general section $s$ is a smooth and of the expected codimension equal to $3\left(\begin{array}{c}k+1 \\ 2\end{array}\right)$. One can also show, using vanishing of cohomology of exterior powers of $\mathcal{S}_{G}^{*}$ that $Z(s)$ is connected if it is of positive dimension. Assume that $k<4$ so that $\operatorname{dim} Z(s)>0$. It is known that the determinant of the tangent bundle of the Grassmannian $G=G(s, n)$ is equal to $c_{1}(G)=n c_{1}\left(S_{G}^{*}\right)$. The determinant of $\Lambda^{2} \mathcal{S}_{G}^{*}$ is equal 
to $(s-1) c_{1}\left(\mathcal{S}_{G}^{*}\right)$. The normal bundle of $Z(s)$ in $G$ is isomorphic to $\mathcal{E}^{\oplus 3}$. Thus, in our case,

$$
c_{1}\left(\mathcal{E}^{\oplus 3}\right)=3 k c_{1}\left(\mathcal{S}_{G}^{*}\right), c_{1}(G)=(2 k+3) c_{1}\left(\mathcal{S}_{G}^{*}\right) .
$$

By adjunction formula,

$$
c_{1}(Z(s))=(3-k) c_{1}\left(\mathcal{O}_{Z(s)}(1)\right),
$$

where $\mathcal{O}_{Z(s)}(1)$ is equal to the restriction of $\operatorname{det} \mathcal{S}_{G}^{*}$ to $Z(s)$. Its sections are hyperplane sections of $Z(s)$ in the Plücker embedding of the Grassmannian. Thus $Z(s)$ is a Fano 3 -fold if $k=1$ or 2 and a K3 surface if $k=3$. If $k=4, \operatorname{dim} Z(s)=0$. The rank of $\mathcal{E}^{\oplus 3}$ is equal to $30=\operatorname{dim} G(5,11)$. Its general section vanishes at the number of points equal to the Chern number $c_{30}\left(\mathcal{E}^{\oplus 3}\right)$. Easy computation shows that this number is equal to 16 .

We know that, for general $F$, the image of $\operatorname{VSP}\left(F ; N_{k}\right)$ in $G(k+$ $\left.1, \mathrm{AP}_{k+1}(F)^{*}\right)$ is smooth, irreducible, and of codimension $3\left(\begin{array}{c}k+1 \\ 2\end{array}\right)$. It is also contained in $Z(s)$, where $s$ is a section of $\mathcal{E}^{\oplus 3}$. We finish the proof if we can show that for a general $F$ the equality takes place. Unfortunately, I don't know how to do this for $k=3,4$ (a proof using sysygies can be found in [12]). So let us assume that $k=1$ or 2 .

Fix a linear space $E$ of dimension $2 k+3$. Suppose we have a 3 dimensional linear subspace $L$ of $\Lambda^{2} E$. We have a natural linear map

$$
\gamma: S^{k+1} L \rightarrow \Lambda^{2 k+2} E,\left(v_{1}, \ldots, v_{k+1}\right) \mapsto v_{1} \wedge \ldots \wedge v_{k+1} .
$$

Let $K$ be the kernel of $\gamma$. For a general $L \in G\left(3, \Lambda^{2} E\right)$, the dimension of $K$ is equal to $\frac{1}{2}(k+3)(k+2)-(2 k+3)=\frac{1}{2} k(k+1)$. Let

$$
\alpha: V \rightarrow L, \quad \beta: S^{k-1} V \rightarrow K
$$

be some linear isomorphisms such that the injection homomorphism $K \hookrightarrow$ $S^{k+1} L$ is isomorphic to the injective homomorphism $\iota: S^{k-1} V \hookrightarrow S^{k+1} V^{*}$. Suppose we can choose $\alpha$ and $\beta$ such that this inclusion corresponds to a totally symmetric tensor

$$
t \in S^{k-1} V^{*} \otimes S^{k+1} V^{*} .
$$

Let $F \in S^{2 k} V^{*}$ be the corresponding homogeneous form on $V$. It is easy to see that the homomorphsism $\iota$ coincides with the homomorphism ap ${ }_{F}^{k-1}$. Then the space $\Lambda^{2 k+2} E \cong E^{*}$ (after a choice of a volume form) and can be identified with the space $\operatorname{AP}_{k+1}(F)^{*}=S^{k+1} V^{*} / \mathrm{ap}_{F}^{k-1}\left(S^{k-1} V\right)$. Moreover, the subspace $L$ of $\Lambda^{2} E$ is identified with the subspace $\mathcal{N}$ defined by the Mukai 2-forms. This can be directly verified by choosing appropriate bases. 
Thus $G\left(k+1, \operatorname{AP}_{k+1}(F)^{*}\right)_{\mathcal{N}}$ is isomorphic to a general subscheme $Z(s)$ and hence the assertion is verified for $F$, hence for any general $F$.

It remains to find $\alpha$ and $\beta$ such that the tensor $t$ is symmetric. This can be done by counting constants. The pairs $(\alpha, \beta)$ are parametrized by $\mathrm{GL}(3) \times \mathrm{GL}\left(\frac{1}{2} k(k+1)\right)$, so depend on $a=9+k^{2}(k+1)^{2} / 4$ parameters. The condition that a tensor $t$ is symmetric is given by

$$
\begin{aligned}
& b=\operatorname{dim} S^{k-1} V^{*} \otimes S^{k+1} V^{*}-\operatorname{dim} S^{2 k} V^{*} \\
= & \frac{(k+3)(k+2)(k+1) k}{4}-(k+1)(2 k+1)
\end{aligned}
$$

linear equations. Thus if $a>b$ we can always choose $(\alpha, \beta)$. If $k=1$, there are no conditions at all. If $k=2$ we have $a=18, b=15$ and it works too. If $k>3, a<b$ and we are in trouble.

\section{Reconstructing a polar polyhedron from its side}

\subsection{The variety of conjugate pairs}

Let $F \in S^{2 k} V$ be a nondegenerate form. Consider the variety of conjugate pairs

$$
\mathrm{CP}(F)=\left\{([l],[m]) \in \mathbb{P} V^{*} \times \mathbb{P} V^{*}: \Omega_{\check{F}}\left(l^{k}, m^{k}\right)=0\right\} .
$$

For any $([l],[m]) \in \mathrm{CP}(F)$ we can find a unique (up to a scalar factor) pair $\left(\Phi, \Phi^{\prime}\right) \in S^{k} V \times S^{k} V$ of anti-polars, i.e.,

$$
D_{\Phi}(F)=l^{k}, D_{\Phi^{\prime}}(F)=m^{k} .
$$

It follows from (2.8) that

$$
\Phi(m)=\Phi^{\prime}(l)=0 .
$$

The equation $\Omega_{\breve{F}}\left(l^{k}, m^{k}\right)=0$ is bi-homogeneous of degree $(k, k)$ in coefficients of $l, m$. For $F$ general enough, it is a smooth $(2 n-1)$-fold $Y$. By adjunction formula, its canonical class $K_{Y}$ is a divisor class corresponding to a hypersurface of bi-degree $(k-n-1, k-n-1)$. So, $Y$ is a Fano variety (i.e., the minus of the canonical class is ample) for $k \leq n$, it is a Calabi-Yau variety if $k=n+1$ (i.e. the canonical class is trivial) and a variety of general type (i.e. the canonical class is ample) if $k>3$.

Consider the projections to the first and the second factor

$$
q: \mathrm{CP}(F) \rightarrow \mathbb{P} V^{*}, \quad q^{\prime}: \mathrm{CP}(F) \rightarrow \mathbb{P} V^{*} .
$$


For any $[l] \in \mathbb{P} V^{*}$ the fibre $q^{-1}([l])$ is a hypersurface of degree $k$ given by the equation $V(\Phi)=0$, where $D_{\Phi}(F)=l^{k}$. The set of points $[l]$ such that $([l],[l]) \in \mathrm{CP}(F)$ is a hypersurface $\mathcal{D}$ of degree $2 k$, it is given by the equation $V(\check{F})=0$.

Let

$$
\widetilde{\operatorname{VSP}}(F ; N)=\left\{([l], \mathcal{P}) \in \mathbb{P} V^{*} \times \operatorname{VSP}(F ; N):[l] \in \mathcal{P}\right\} .
$$

The projection to the second factors is a finite map of degree $N$ ramified over the complement of $\operatorname{VSP}(F ; N)^{o}$. If we choose the Hilbert scheme compactification $\operatorname{VSP}(F ; N)$, then $\widetilde{\operatorname{VSP}}(F ; N)$ is the restriction of the universal scheme over $\operatorname{VSP}(F ; N)$.

Consider the first projection

$$
p: \widetilde{\operatorname{VSP}}(F ; N) \rightarrow \mathbb{P} V^{*} .
$$

Its fibre over a line $V(l)$ is the set of (generalized) polar polyhedra with the line $V(l)$ as its side.

4.2. The case $(n, d, N)=(2,2,3)$.

Let $([l],[m]) \in \mathrm{CP}(F),[l] \neq[m]$, and $\left(\Phi, \Phi^{\prime}\right)$ be the corresponding pair of anti-polars (4.1). Let $[n]$ be the the intersection point of the corresponding lines $V(\Phi)$ and $V\left(\Phi^{\prime}\right)$. Obvioulsy $[n] \neq[l],[n] \neq[m]$ since $[l]$ and $[m]$ are not self-conjugate. For any linear forms $L_{1}, L_{2}$ such that $L_{1}(l)=L_{2}(m)=0$, the conic $L_{1} \Phi_{1}+L_{2} \Phi_{2}$ vanishes at the points $[l],[m],[n]$ and obviously belongs to $\mathrm{AP}_{2}(F)$. The dimension of the space of such conics is equal to 3 . By Lemma 3.1, we obtain that $\{[l],[m],[n]\}$ is a polar triangle of $F$. Thus we obtain a regular map

$$
f: \mathrm{CP}(F)^{o} \rightarrow \widetilde{\operatorname{VSP}}(F ; 3),([l],[m]) \mapsto([n],\{[l],[m],[n]\})
$$

where

$$
\mathrm{CP}(F)^{o}=\mathrm{CP}(F) \backslash(\mathrm{CP}(F) \cap \text { diagonal }) .
$$

One can show that this map extends to the blow-up $\widehat{\mathrm{CP}(F)}$ of $\mathrm{CP}(F)$ along its intersection with the diagonal. It defines a regular map of degree 3

$$
\widehat{\mathrm{CP}(F)} \rightarrow \widetilde{\mathrm{VSP}}(F ; 3) \text {. }
$$

The involution $\iota:([l],[m]) \mapsto([m],[l])$ extends to $\widehat{\mathrm{CP}(F})$ and the quotient $\widehat{\mathrm{CP}(F)} / \iota$ is a nonsingular hypersurface in the Hilbert scheme $(\mathbb{P} V)^{[2]}$. The 
composition of the map $f$ with the projection $\widetilde{\operatorname{VSP}}(F ; 3) \rightarrow \operatorname{VSP}(F ; 3)$ defines a regular map of degree 2

$$
\widehat{\mathrm{CP}(F)} / \iota \rightarrow \operatorname{VSP}(F ; 3) .
$$

It is unramified over $\operatorname{VSP}(F ; 3)^{o}$. In particular, we see that any polar triangle of $F$ is uniquely reconstructed from its two sides, and any line occurs as a side of one of polar triangles.

Remark 4.1. It is easy to see that the universal cover of $\operatorname{VSP}(F, 3)^{\circ}$ is isomorphic to the algebraic group $\mathrm{SO}(3)$. The fundamental group is isomorphic to its Weyl group $G=2^{2} \rtimes S_{3}$ of order 24 . The quotient by the normal subgroup $2^{2}=(\mathbb{Z} / 2)^{2}$ is the variety of ordered polar triangles of $F$. The quotient by the group $2^{2} \rtimes 2$ is isomorphic to $\mathrm{CP}(F)^{o}$.

4.3. The case $(n, d, N)=(2,4,6)$.

Let $([l],[m]) \in \operatorname{CP}(F)^{o}$ and $\Phi, \Phi^{\prime}$ be their anti-polars of degree 2. Assume that $\Phi, \Phi^{\prime}$ have no common irreducible components. Consider the linear subspace $L$ of $S^{4} V$ formed by forms $A \Phi+A^{\prime} \Phi^{\prime}$, where $A, A^{\prime} \in S^{2} V$ and $A(l)=0, A^{\prime}(m)=0$. Since

$$
D_{A \Phi+A^{\prime} \Phi^{\prime}}(F)=D_{A}\left(D_{\Phi}(F)\right)+D_{A^{\prime}}\left(D_{\Phi^{\prime}}(F)\right)=D_{A}\left(l^{2}\right)+D_{A^{\prime}}\left(m^{2}\right)=0
$$

we see that

$$
L \subset \mathrm{AP}_{4}(F) .
$$

Consider the map $\left(A, A^{\prime}\right) \mapsto A \Phi+A^{\prime} \Phi^{\prime}$. The kernel of this map consists of pairs $\left(A, A^{\prime}\right)$ such that $A \Phi=-A^{\prime} \Phi^{\prime}$. Since no irreducible component of $\Phi^{\prime}$ divides $\Phi$, we obtain that $\Phi^{\prime}$ divides $A$ and hence $A=c \Phi^{\prime}, A=-c \Phi$. Thus the kernel of the map is one-dimensional and is spanned by $\left(\Phi^{\prime},-\Phi\right)$. From this we obtain that $\operatorname{dim} L=9$ and hence coincides with $\operatorname{dim} L_{4}\left(\mathbb{P} V^{*}, Z\right)$, where $Z$ is the closed subscheme formed by the points $[l],[m]$ and the common zeroes of the conics $V(\Phi), V\left(\Phi^{\prime}\right)$. Thus, by Lemma 3.1, $Z$ is a polar hexagon of $F$ (a generalized one if $V(\Phi)$ does not intersect transversally $\left.V\left(\Phi^{\prime}\right)\right)$.

Thus, as in the previous case we obtain a rational map.

$$
\widehat{\mathrm{CP}(F)} / \iota-\rightarrow \operatorname{VSP}(F ; 6) .
$$

It is of degree $15=\left(\begin{array}{l}6 \\ 2\end{array}\right)$. Thus any polar hexagon of $F$ is uniquely reconstructed from its two sides, and any line occurs as a side of one of polar hexagons. 
Remark 4.2. Under the regular map $f: \mathrm{CP}(F)^{o} \rightarrow \widetilde{\mathrm{VSP}}(F ; N)$ the fibres of any of the two projections $\mathrm{CP}(F)^{o} \rightarrow \mathbb{P} V^{*}$ are mapped isomorphically to the fibres of the projection $\widetilde{\mathrm{VSP}}(F ; 6) \rightarrow \mathbb{P} V^{*}$. This implies that a general point of $\operatorname{VSP}(F ; 6)$ lies on 6 rational curves. It can be shown that these curves are conics in the anti-canonical embedding $\operatorname{VSP}(F ; 6) \subset G(3,7) \subset$ $\mathbb{P}^{20}$ (see $[9]$ ). It is known that the variety of conics on the Fano variety $\operatorname{VSP}(F ; 6)$ is isomorphic to the projective plane. According to [10], this plane can be identified with $\mathbb{P} V^{*}$ and the six conics through a point $x$ represent the sides of the polar hexagon corresponding to the point $x$.

4.4. The case $(n, d, N)=(2,6,10)$.

The situation here is a little different from the previous cases.

Theorem 4.3. Let $F$ be a general homogeneous polynomial of degree 6 in 3 variables. Any line in $\mathbb{P}^{2}$ can be realized as a side of a polar polyhedron of F. For a general line, there are at most two polyhedra with the same side. For a general pair of lines there are at most one polyhedron which has these lines as its sides.

Proof. We have to show that the projection $\widetilde{\operatorname{VSP}}(F ; 10) \rightarrow \mathbb{P}^{2}$ is surjective and of degree 2 . Note that it cannot be of degree 1 since $\operatorname{VSP}(F ; 10)$ is a $\mathrm{K} 3$ surface and hence is not unirational. The assertion follows from the following fact which can be found in [2]: There are at most two polar polyhedra of $F$ which share a common side $[l]$. Since I do not understand Dixon's proof I suggest my own proof of the statement. Suppose

$$
F=l^{6}+l_{1}^{6}+\ldots+l_{9}^{6}=\lambda l^{6}+m_{1}^{6}+\ldots+m_{9}^{6} .
$$

Choose $\Phi, \Phi^{\prime} \in S^{3} V$ such that $V(\Phi)$ vanishes at the points $\left[l_{i}\right]$ and $V\left(\Phi^{\prime}\right)$ vanishes at the points $\left[m_{i}\right]$. We can normalize them to assume that $\Phi(l)=$ $\lambda \Phi^{\prime}(l)$. Then

$$
D_{\Phi}(F)=D_{\Phi^{\prime}}(F)=120 \Phi(l) l^{3},
$$

hence $D_{\Phi-\Phi^{\prime}}(F)=0$. Since $F$ is nondegenerate, $\operatorname{AP}_{3}(F)=\{0\}$, and thus $\Phi=\Phi^{\prime}$. The equality

$$
(\lambda-1) l^{6}=\left(l_{1}^{6}+\ldots+l_{9}^{6}\right)-\left(m_{1}^{6}+\ldots+m_{9}^{6}\right),
$$

implies that $(\lambda-1) D_{\Phi}\left(l^{6}\right)=0$. If $\lambda \neq 1$ we get $D_{\Phi}(l)=0$ and hence $D_{\Phi}(F)=0$ contradicting the nondegeneracy of $F$. Thus $\lambda=1$, and

$$
l_{1}^{6}+\ldots+l_{9}^{6}=m_{1}^{6}+\ldots+m_{9}^{6} .
$$

Since $l_{1}^{6}, \ldots, l_{9}^{6}, m_{1}^{6}, \ldots, m_{9}^{6}$ span a subspace $L \subset S^{6} V^{*}$ of dimension $\leq 17$, the dimension of the orthogonal complement of $L$ in $S^{6} V$ is of dimension 
$\geq 28-17=11$. The space of forms divisible by $\Phi$ is of dimension 10 . Thus there exists a form $G \in S^{6} V$ not vanishing on the cubic curve $V(\Phi)$ but vanishing at the 18 points $\left[l_{i}\right],\left[m_{i}\right]$ on $V(\Phi)$. Assume there is a third polar polyhedron of $F$ with a side $[l]$. We have

$$
F=l^{6}+n_{1}^{6}+\ldots+n_{9}^{6}=l^{6}+l_{1}^{6}+\ldots+l_{9}^{6}=l^{6}+m_{1}^{6}+\ldots+m_{9}^{6},
$$

and all 27 points $\left[l_{i}\right],\left[m_{i}\right],\left[n_{i}\right]$ 's lie on the cubic $V(\Phi)$. Similar to above we get a form $G^{\prime}$ of degree 6 vanishing at $\left[m_{i}\right],\left[n_{i}\right]$ 's and a form $G^{\prime \prime}$ of degree 6 vanishing at $\left[l_{i}\right],\left[n_{i}\right]$ 's. This easily implies that the divisors $\sum\left[l_{i}\right], \sum\left[m_{i}\right]$, $\sum\left[n_{i}\right]$ of degree 9 on the cubic $V(\Phi)$ are linear equivalent. Also we see that the divisor $\sum 2\left[l_{i}\right]$ is cut out by a sextic $C$. It can be chosen to have at least double points at $\left[l_{i}\right]$ 's (see [4], Lemma 4.4). The pencil of sextics formed by $C$ and $V\left(\Phi^{2}\right)$ is an Halphen pencil of elliptic sextics. Choose a member $V(K)$ of this pencil passing through the point $[l]$. Then we obtain that $D_{K}(F)=0$, hence $\operatorname{AP}_{6}(F)$ contains a sextic with 9 double points.

To summarize, we obtain that for a general point $[l] \in \mathbb{P} V^{*}$, the variety $\operatorname{VSP}(F ; 10)$ contains a polar polyhedron $[l],\left[l_{1}\right], \ldots,\left[l_{9}\right]$ such that the 9 points $\left[l_{i}\right]$ form the set of base points of an Halphen pencil of curves of degree 6 . We call such a polyhedron an Halphen polyhedron. It is easy to see that the locus of such sets in $\left(\mathbb{P} V^{*}\right)^{(9)}$ is of codimension 1. Thus, for general $F$, the surface $\operatorname{VSP}(F ; 10)$ intersects this divisor along a variety of dimension $\leq 1$ (an easy count of constants shows that it cannot be contained in the divisor). Its pre-image in $\widetilde{\mathrm{VSP}}(F ; 10)$ is of dimension $\leq 1$. Its image under the projection $q: \widetilde{\operatorname{VSP}}(F ; 10) \rightarrow \mathbb{P} V^{*}$ is of dimension $\leq 1$. Thus for a general $[l]$, the fibre $q^{-1}([l])$ cannot not contain an Halphen polyhedron. This contradiction proves the first assertion.

Let us prove the second assertion. Assume that

$$
F=l^{6}+m^{6}+l_{1}^{6}+\ldots+l_{8}^{6}=\lambda l^{6}+\mu m^{6}+m_{1}^{6}+\ldots+m_{8}^{6} .
$$

Let $\Phi$ be the cubic anti-polar of $l$, i.e. $D_{\Phi}(F)=l^{3}$ and similarly let $\Phi$ be the cubic anti-polar of $m$. By the nondegeneracy of $F$, the anti-polars are unique, hence $V(\Phi)$ is the cubic containing the points $[m],\left[l_{1}\right], \ldots,\left[l_{8}\right]$ and $V\left(\Phi^{\prime}\right)$ is the cubic containing the points $[l],\left[l_{1}\right], \ldots,\left[l_{8}\right]$. Thus the points $\left[l_{1}\right], \ldots,\left[l_{8}\right]$ are among 9 intersection points of the cubics $V(\Phi)$ and $V\left(\Phi^{\prime}\right)$. Using the second polar polyhedron, we obtain that the points $\left[m_{1}\right], \ldots,\left[m_{8}\right]$ are in the same set of 9 points. This implies that either the sets $\left[l_{1}\right], \ldots,\left[l_{8}\right]$ and $\left[m_{1}\right], \ldots,\left[m_{8}\right]$ coincide or have a common set of 7 points. In the first 
case we are done. In the second case we may assume that

$$
F=l^{6}+m^{6}+l_{1}^{6}+\ldots+l_{7}^{6}+l_{8}^{6}=\lambda l^{6}+\mu m^{6}+\lambda_{1} l_{1}^{6}+\ldots+\lambda_{7} l_{7}^{6}+m_{8}^{6} .
$$

Let $V(G)$ be a cubic passing through the points $[l],[m],\left[l_{1}\right], \ldots,\left[l_{7}\right]$. We have $D_{G}(F)=120 G\left(l_{8}\right) l_{8}^{3}=120 G\left(m_{8}\right) m_{8}^{3}$. This implies $\left[l_{8}\right]=\left[m_{8}\right]$ and proves the assertion.

Remark 4.4. Let $F$ be a general form of degree 6 in 3 variables. It follows from the proof of the previous theorem that the variety $\widetilde{\mathrm{VSP}}(F, 10)$ is birationally isomorphic to a double cover of projective plane. It would be interesting to find the degree of the branch curve.

4.5. The case $(n, d, N)=(3,4,10)$.

Let $F$ be a general quartic form in 4 variables. We have already remarked that this is one of the cases where the Waring rank reaches its minimum equal to 10 . It is not even known the birational type of the variety $\operatorname{VSP}(F ; 10)$. The arguments similar to ones used in the previous section show that a polar polyhedron of $F$ can be reconstructed in at most two ways from a pair of its two faces (i.e. a pair of conjugate planes). Using the method of syzygies from [12], Michael Sagraloff [15] proves that it is exactly two ways. Note that this result was claimed by A. Dixon [2] and Reye [13] erroneously claimed that the reconstruction can be done in one way.

\section{References}

[1] A. Hirschowitz, J. Alexander, Polynomial interpolation in several variables. J. of Alg. Geom 4 (1995), 201-222.

[2] A. C. Dixon, The canonical forms of the ternary sextic and quaternary quartic. London Math. Soc. Proc. (2) 4 (1906), 223-227.

[3] I. Dolgachev, V. Kanev, Polar covariants of plane cubics and quartics. Adv. in Math. 98 (1993), 216-301.

[4] I. Dolgachev, Lecture on Invariant Theory. LMS Lecture Notes Series 296, Cambridge University Press, 2003.

[5] J. Fogarty, Algebraic families on an algebraic surface. Amer. J. Math. 90 (1968), 511-521.

[6] W. Fulton, Intersection Theory. 3rd Edition, Birkhäuser, 2000.

[7] A. Iarrobino, V. Kanev Power Sums, Gorenstein Algebras and Determinantal Loci. Lecture Notes in Math. 1721, Springer, 1999. 
[8] A. Illiev, K. Ranestad, K3 surfaces of genus 8 and varieties of sums of powers of cubic fourfolds. Trans. A.M.S. 353 (2001), 1455-1468.

[9] F. Melliez, K. Ranestad, Degenerations of (1,7)-polarized abelian surfaces. arXiv. math. AG/0309306.

[10] S. Mukai, Fano threefolds. In "Complex Projective Geometry", LMS Lecture Notes Series, Cambridge University Press, 1992, 255-263

[11] S. Mukai, Polarized K3 surfaces of genus 18 and 20. In "Complex Projective Geometry", LMS Lecture Notes Series, Cambridge University Press, 1992, 264-276.

[12] K. Ranestad, F.-O. Schreier, Varieties of sums of powers. J. Reine Angew. Math. 525 (2000), 147-181.

[13] Th. Reye, Darstellung quaternären biquadratischer Formen als Summen von zehn Biquadraten. J. Reine Angew. Math. 58, (1874), 123-129.

[14] H. W. Richmond, On canonical forms. Quart. J. Math. 33 (1902), 331-340.

[15] M. Sagraloff, Quartiken als Summe von Potenzen in $\mathbb{P}^{3}$. Diplomarbeit, Univ. Bayreuth, 2002.

Igor V. Dolgachev

Department of Mathematics

University of Michigan

Ann Arbor, Michigan 49109

USA

e-mail: idolga@umich.edu

Lecture held in the Seminario Matematico e Fisico on October 15, 2003

Received: April, 2004 